\title{
Remote Cardiac Rehabilitation in Older Cardiac Disease: A Randomized Case Series Feasibility Study
}

\author{
Masakazu Saitoh $^{\mathrm{a}, \mathrm{h}}\left({ }^{0}\right.$, Tetsuya Takahashi ${ }^{\mathrm{a}, \mathrm{h}}$, Tomoyuki Morisawa ${ }^{\mathrm{a}}$, Akio Honzawa $^{\mathrm{b}}$, \\ Miho Yokoyama ${ }^{\mathrm{b}, \mathrm{c}}$, Abidan Abulimitic, d, Nobuyuki Kagiyama ${ }^{\mathrm{c}, \mathrm{e}}$, \\ Takatoshi Kasai ${ }^{c,}$ e, Tohru Minaminoc, Tohru Asaif, \\ Toshiyuki Fujiwara ${ }^{a, g}$, Hiroyuki Daida ${ }^{\text {a, }}$ c
}

\begin{abstract}
Background: Cardiac rehabilitation (CR) in the long term is fundamental to achieve better outcomes. However, most patients may find it difficult to maintain healthy lifestyle behavior after ambulatory $\mathrm{CR}$ program. We aimed to investigate the feasibility on remote CR support program (Remote-CR) in older patients with cardiac disease.

Methods: Between October 2020 and February 2021, we enrolled older patients with cardiac disease after at least over 5 months of ambulatory $\mathrm{CR}$ program. The study was conducted as a randomized controlled case series to compare Remote-CR vs. center-based CR (CB$\mathrm{CR}$ ) after ambulatory $\mathrm{CR}$ in older patients with cardiac disease. The core component of Remote-CR is a web application, which evaluates vital sign and receives feedback through real-time video consultation once a week. We evaluated the satisfaction of Remote-CR, and impact of Remote-CR on adherence to exercise and physical activity management. Moreover, we assessed the change in functional status using handgrip strength, usual gait speed, short physical performance battery (SPPB), and Kihon checklist (KCL) before the intervention,
\end{abstract}

Manuscript submitted December 21, 2021, accepted December 28, 2021

Published online January 10, 2022

aDepartment of Physical Therapy, Faculty of Health Science, Juntendo University, Tokyo, Japan

${ }^{\mathrm{b}}$ Cardiovascular Rehabilitation and Fitness, Juntendo University Hospital, Tokyo, Japan

'Department of Cardiovascular Biology and Medicine, Juntendo University Graduate School of Medicine, Tokyo, Japan

${ }^{\mathrm{d} S}$ Sportology Center, Juntendo University Graduate School of Medicine, Tokyo, Japan

eDepartment of Digital Health and Telemedicine R\&D, Faculty of Health Science, Juntendo University, Tokyo Japan.

fDepartment of Cardiovascular Surgery, Juntendo University Graduate School of Medicine, Tokyo, Japan

${ }^{g}$ Department of Rehabilitation Medicine, Juntendo University Graduate School of Medicine, Tokyo, Japan

${ }^{\mathrm{h}}$ Corresponding Author: Masakazu Saitoh and Tetsuya Takahashi, Department of Physical Therapy, Faculty of Health Science, Juntendo University, Tokyo, Japan. Email: m.saito.tl@juntendo.ac.jp and te-takahashi@juntendo.ac.jp

doi: https://doi.org/10.14740/cr1346 at the end of the 4-week intervention, and cardiovascular-related hospital discharge within 6 months after completion of ambulatory CR.

Results: Eleven patients were enrolled (six for Remote-CR and five for CB-CR). All participants completed the intervention without serious adverse event in each group. After the on-site initial remote system set up, all the patients completed the Remote-CR session without on-site assistance. Patients' satisfaction with Remote-CR was generally high. Remote-CR helps to maintain healthy lifestyle behavior. In addition, Remote-CR and CB-CR seem to be equally successful in maintaining functional status and preventing cardiovascular-related hospital readmission.

Conclusions: This trial suggested the Remote-CR comprising a hybrid of information and communication technology and strategy for health behavior change may be a feasible and safe intervention option in selected older patients with cardiac disease after completion of ambulatory CR.

Keywords: Remote; Cardiac rehabilitation; Older; Functional status; Prognosis

\section{Introduction}

Cardiac rehabilitation (CR) is a multidisciplinary, comprehensive program to improve exercise capacity and quality of life $(\mathrm{QoL})$, and reduce risk of hospital readmission [1]. CR is usually provided through group-based program in CR center, which induces therapeutic exercise and self-management education on secondary prevention. Maintaining unsupervised CR in the long term even after completion of outpatients CR program is fundamental to achieve better outcomes. However, its long-term benefits are less promising because most patients may find it difficult to maintain CR [2].

Currently, CR program should provide a variety of delivery option for patients according to their need or preferences. The remote systems using the internet enable patients to participate in the home setting [3], which has contributed to improving CR adherence [2]. Several systematic reviews also 
suggested that real-time remote exercise-based CR has comparable favorable effects on functional status, QoL, and reduced morbidity as compared with center-based CR (CB-CR) [4-6]. However, there are few studies to examine the effect of remote CR support program (Remote-CR), which evaluates vital sign and provides feedback through real-time video consultation due to strategy for health behavior change in older patients with cardiovascular disease, particularly in Japan [7].

Functional decline or deterioration in physical activity was observed after CR completion in older subjects, even in a short period of time $[8,9]$. Marchionni et al suggested that continuously reinforced intervention after CR completion maintained long-term benefits [10]. To our knowledge, there is no study to investigate the feasibility on Remote-CR in adherence of unsupervised $\mathrm{CR}$ and maintaining functional status after $\mathrm{CR}$ completion in older patients with cardiac disease. This study aimed to assess whether Remote-CR after completion of ambulatory CR is able to assist health behavior change, and is effective in maintaining their functional status and short-term prognosis after CR completion in older patients with cardiovascular disease.

\section{Materials and Methods}

\section{Participants}

The study was conducted at Juntendo University Hospital, Tokyo, Japan. Study subjects were older patients enrolled in ambulatory $\mathrm{CR}$ program. Inclusion criteria were as follows: 1) aged 65 years or older; 2) stable adult patients with cardiac disease, defined as completed ambulatory CR program for at least over 5 months without adverse cardiac events; 3 ) internet access at home; and 4) patients with family or caregiver to support remote CR. Exclusion criteria were as follows: 1) unable to complete the basic course of treatment with poor adherence or inability to follow-up; and 2) severe cognitive impairment or lack of digital literacy which prohibits them to operate Remote-CR system.

The study was a randomized clinical trial (RCT) performed according to the CPONSORT statement [11]. The RCT was conducted from October 20, 2020 to February 30, 2021, in the CR Center at the Juntendo University Hospital, Japan. The study was conducted as an open label, randomized, controlled study. Patients were randomized with an allocation ratio of $1: 1$, based on a computer-generated list of random numbers. Allocation concealment was ensured since allocation information was protected in opaque sealed envelopes and kept by an investigator who was involved in patient recruitment. The trial was approved by the ethic committee of Department of Physical Therapy, Faculty of Health Science, Juntendo University (20-156) and registered with the University Hospital Medical Information Network (UMIN) clinical trial registry (UMIN 000042275), and the study was conducted in accordance with the principles of the Declaration of Helsinki. All participants gave written consent to participation after explaining the study procedures. We have done sample size power calculation, and a sample size of 46 patients was chosen based on the recommended methods. The present was terminated before it had reached a sufficient sample size due to the spread of coronavirus disease 2019 (COVID-19) and interruption of ambulatory $\mathrm{CR}$ program due to state of emergency and quarantine.

\section{Study interventions}

The patients received Remote-CR program and learned how to use the tablet computer (iPad, Apple, Cupertino, CA) and remote real-time telemedicine system (Nipro HeartLine ${ }^{\mathrm{TM}}$, NIPRO). Videoconference was performed through Nipro HeartLine ${ }^{\mathrm{TM}}$, which also enabled health providers to monitor the health condition of patient's vital sign in real time on a tablet device. Participants were asked to encourage unsupervised exercise using original exercise video file detailing the aerobic, resistance, balance, and flexibility exercises, and record their exercises or pedometer-assessed daily steps in a logbook. The electrocardiograph (Duranta, ZAIKEN, Japan), pulse oximeter (MightySatTMRx, Masimo, USA) and electronic sphygmomanometer (NBP-1BLE, NIPROm Japan) were used with a Bluetooth connection to a tablet computer, and data were recorded in the remote monitoring system. Patients were asked to continue unsupervised exercises for three or more times a week for 4 weeks after completing the 12 weeks of ambulatory CR program. The exercise program was $20-30 \mathrm{~min}$ at Borg scale of 11 - 13. Patients and family or caregivers were asked to fill the exercise diary after each exercise training, pedometer-assessed daily steps, and disease management status (body weight, leg edema, fatigue, blood pressure, heart rate, short of breath, oxygen saturation, pedometer trend, appetite, healthy diet, medication adherence, and daily stress). The physical therapists with more than 20 years of professional experience were responsible for contacting patients via a remote real-time monitoring system weekly. In addition, physical therapists checked handwritten logbook including exercise diary, pedometer-assessed daily steps, and disease management status through the display of videoconference, and provided feedback to the patients (Fig. 1). The cost for telemedicine system is the US dollars (USD) and Eros (EUR) at the exchange rate on October 20, when this study started.

In the CB-CR group, patients visited CR Center, Juntendo University Hospital for 1 - 2 times a week for 4 weeks after completing the 12 weeks of ambulatory CR program. CB-CR program included individualized supervised progressive aerobic exercise and resistance exercise. In addition, secondary prevention education or guidance was instructed by health fitness programmer, nurses, or physician certified from the Japanese Association of Cardiac Rehabilitation (JACR) [1]. All participants were encouraged to home-based aerobic exercise for 20 - $30 \mathrm{~min}$ and resistance exercise at Borg scale of $11-13$ for 1 - 2 times a week in addition to CB-CR session for total of at least three times a week.

\section{A survey for feasibility}

In this study, the following four questions were assessed on satisfaction and adherence to exercise or physical activity management with Remote-CR program, and a 5-point Likert 


\section{Component of comprehensive cardiac rehabilitation}

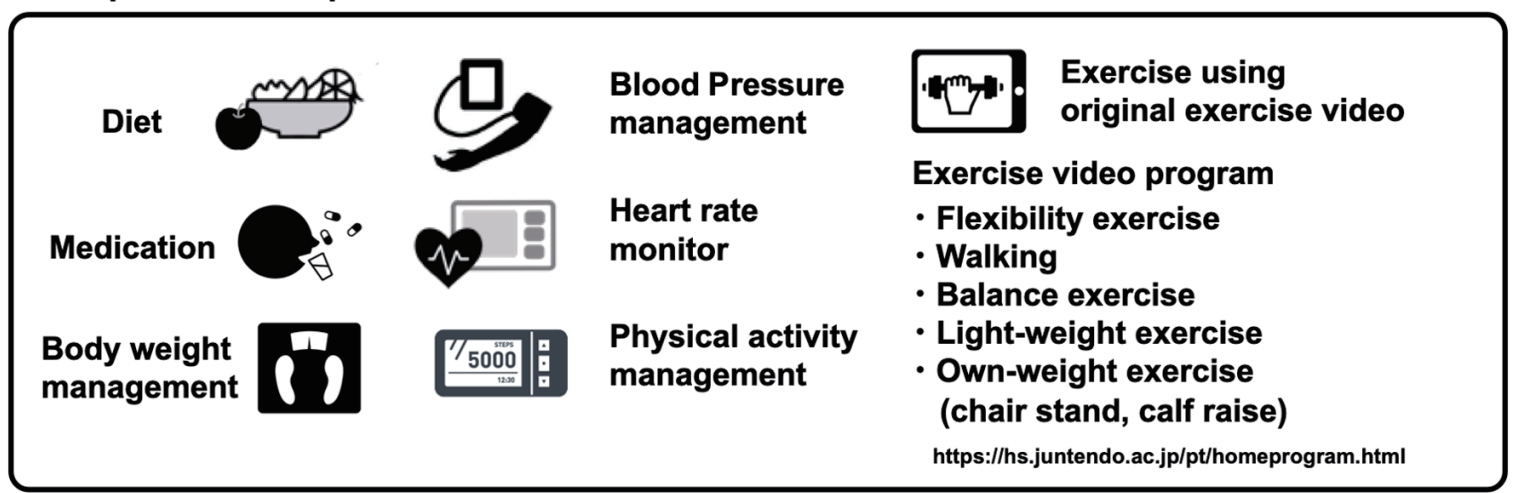

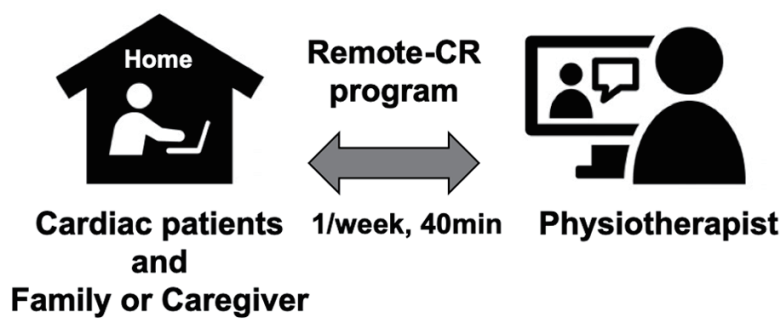

Check List

Vital sign: Heart rate, Blood pressure, Body weight

Symptom: Short of breath, edema, fatigue, appetite

Self care: Exercise, Physical activity, Diet,

Medication

Family or Caregiver

Figure 1. Schema of Remote-CR. Patients who participated in Remote-CR program were given tablet PC guides for exercise program staff; after completion of ambulatory $\mathrm{CR}$, remote consultation program was provided to them by trained physiotherapist, once a week for 4 weeks. Remote-CR: remote cardiac rehabilitation support program.

scale ranging from 1 (strongly disagree) to 5 (strongly agree): "Remote-CR was an acceptable way to receive a CR program", "I have more time for exercise", "I have increased my physical activity", and frequency of video-based exercise application use with 4-point Likert scale ranging from 1 (never) to 4 (at least three times a week): "How many days per week did you use the video-based exercise application?"

\section{Functional assessment}

We assessed short physical performance battery (SPPB), handgrip strength, gait speed [12], and Kihon checklist (KCL), which is a self-reported comprehensive health checklist for evaluation of vulnerable to frailty. All baseline measures were collected during the ambulatory CR program before randomization. Follow-up physical functional assessment was collected by trained physical therapist, non-blinded assessors according to standardized protocols after completing the 4-week intervention.

\section{Handgrip strength}

Isometric handgrip strength was measured using the Jamar hand dynamometer, which measured strength in kilograms with a precision of $0.1 \mathrm{~kg}$. Patients were instructed to sit on a straight-backed chair with both feet placed flat on the floor, shoulder adducted and neutrally rotated, elbow flexed at $90^{\circ}$, and forearm and wrist in neutral position, as recommended by Roberts et al [13]. Grip strength was measured twice per hand in each of the two handle positions. Patients were instructed to apply maximum power for $3 \mathrm{~s}$ and work at maximum effort each trial.

\section{Usual gait speed}

During the 5-m walk test, time was recorded, and gait speed was determined in meter per second. Use of cane was acceptable. Participations were asked to walk $5 \mathrm{~m}$ at their comfortable speed without overexerting. Using a digital stopwatch that records time within $0.01 \mathrm{~s}$, timing was manually started at the "go" instruction and stopped when the patients crossed the 5-m line [14].

\section{$S P P B$}

The SPPB is a highly standardized geriatric physical functioning test that consists of tests for balance, gait, strength, and endurance [12]. The standing balance test included side-by-side, semi-tandem, and tandem standing, and patients were timed until they lost balance or after $10 \mathrm{~s}$. The gait test assessed the time taken to walk $4 \mathrm{~m}$ and was performed at patient's usual pace. The five-time chair-standing test, a pre-test, was also performed, wherein patients were asked to fold their arms across their chest and stand up from the chair. If the pre-test was successful, patients were asked to rise from the chair five times consecutively as quickly as possible. Each of the three subtests of SPPB was scored from 0 to 4 and summed for an SPPB score of 0 to 12 points. Higher scores indicated better physical function. 


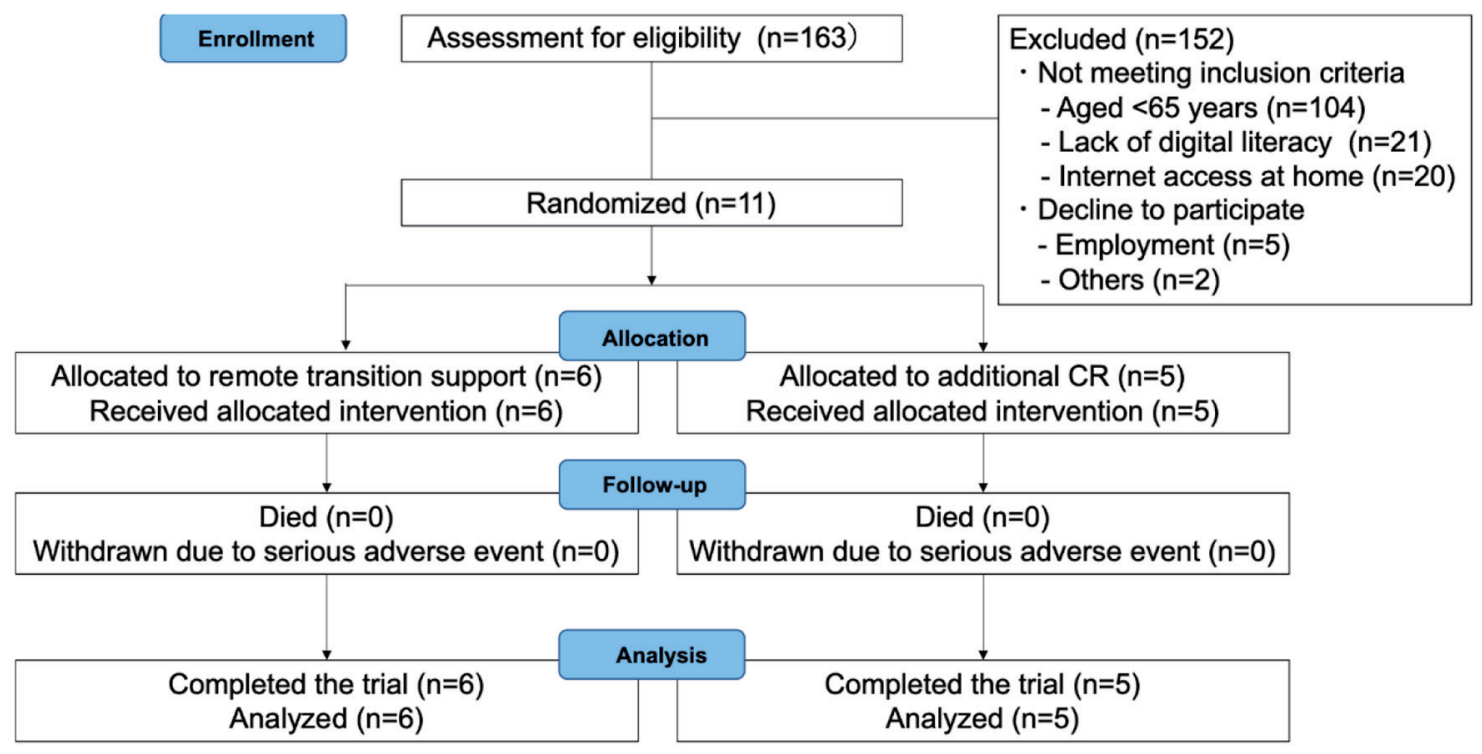

Figure 2. Trial flow chart. CR: cardiac rehabilitation.

$K C L$

The patients were also provided with KCL, which is a selfreported comprehensive health checklist for evaluation of vulnerable to frailty $[15,16]$. The KCL consists of 25 items (yes/ no) divided into seven categories: instrumental activities of daily living, physical strength, nutrition, eating, socialisation, memory, and mood, each item is scored from 0 to 1 point, and the sum of all indices can be from 0 to 25 . A total score of $\geq 8$ points is classified as indicating frailty [16].

\section{Outcomes}

Our outcomes were feasibility of Remote-CR, change in functional status, and cardiovascular-related hospital discharge within 6 months after completion of ambulatory CR between study groups.

\section{Statistical analysis}

Clinical characteristics were summarized using medians with interquartile range (IQR) or proportions. To assess for differences of baseline data between two groups, the following tests were used: Fisher's exact test for dichotomy variables, MannWhitney test for non-normally distributed continuous variables, and Chi-square test for categorical variables. Within-group difference of changes in functional status between baseline and 4 weeks after intervention periods used Wilcoxon signed-rank test. To compare change in functional status between the two groups, the absolute difference during the intervention periods was calculated, and a Mann-Whitney test was performed. Statistical analysis was conducted using SPSS version 23.0 for Windows (IBM Corp., Armonk, New York).

\section{Results}

\section{Participant flow}

One hundred sixty-three patients were screened in the trial periods. Figure 2 reports patients not meeting inclusion criteria and patients meeting the exclusion criteria at baseline. Recruitment rates were extremely low due to patients not meeting the inclusion criteria due to no internet access at home. In addition, feasibility of the study was complicated by the spread of COVID-19. Included patients were allocated to Remote-CR group $(n=6)$ and CB-CE group $(n=5)$, and 11 (100\%) completed the follow-up assessment. All participants completed one to two exercises during intervention periods in each group. There was no serious adverse event in both Remote-CR and CB-CR groups during 4-week intervention periods.

\section{Baseline data}

The baseline demographic and clinical characteristics of the patients in each group are summarized in Table 1. The median age was 73 years (range: 68 - 78 years), and three were female. A positive baseline frailty score was found in one patient in the CB-CR group (9\%). A positive baseline low muscle strength was found in three patients in the Remote-CR group and in three in CB-CR group (55\%). No patient had baseline slow gait speed in each group. We found no significant differences in baseline demographic and clinical characteristics between the two groups. At baseline, no between group difference was found in the handgrip strength $(27.80(22.75$ - 33.00) vs. 33.00 $(25.75-34.50) \mathrm{kg})$, gait speed $(3.345(2.303-3.788)$ vs. 3.080 $(2.980-3.680) \mathrm{s})$, five-time chair stand test $(8.220(7.053-$ $9.598)$ vs. $7.000(6.535-7.625) \mathrm{s})$, SPPB score (11.50 (10.75 
Table 1. Baseline Characteristics

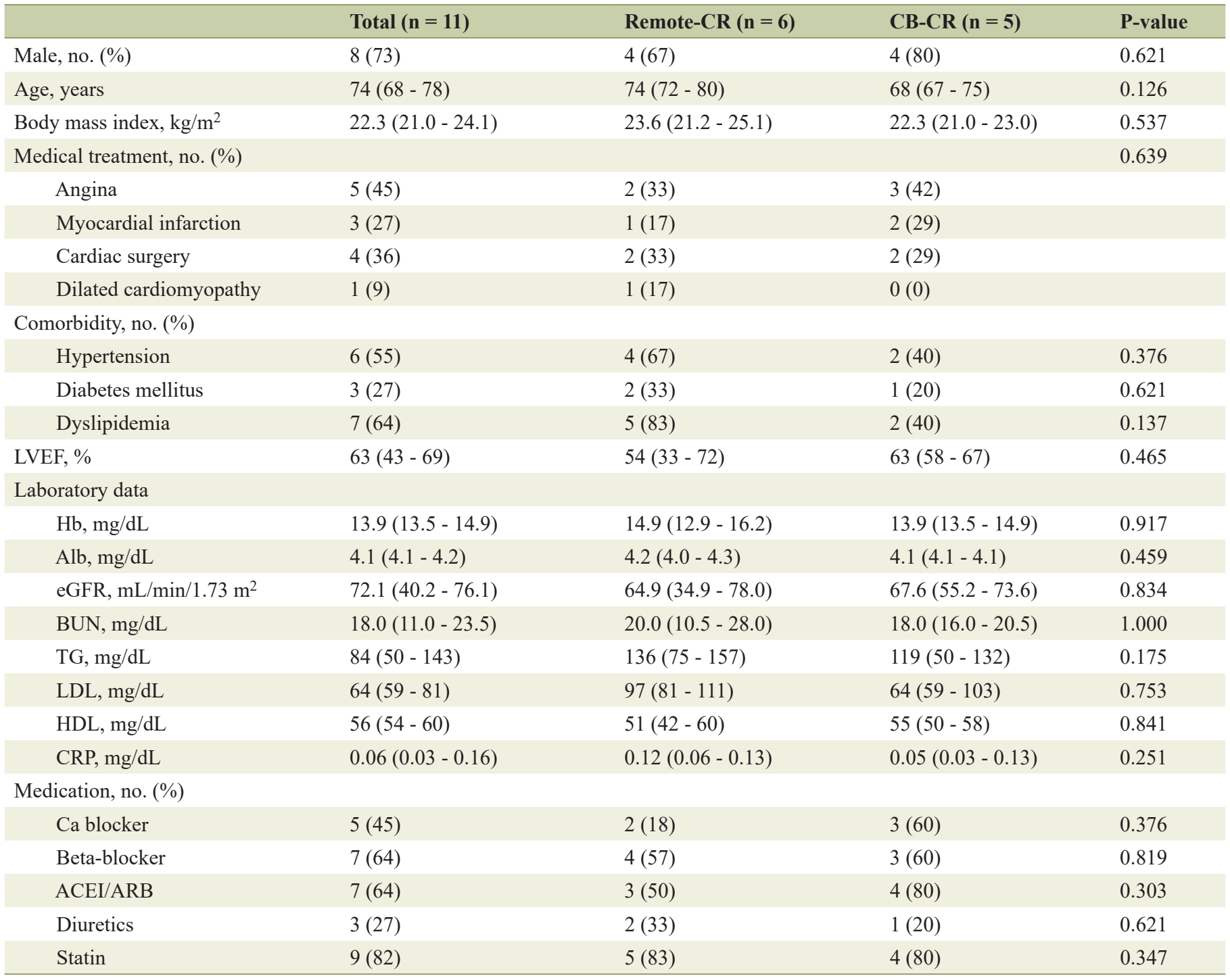

CR: cardiac rehabilitation; LVEF: left ventricular ejection fraction; $\mathrm{Hb}$ : hemoglobin; eGFR: estimated glomerular filtration rate; BUN: blood urine nitrogen; Alb: albumin; TG: triglyceride; LDL: low-density lipoprotein; HDL: high-density lipoprotein; CRP: C-reactive protein; Ca: calcium; ACEI: angiotensin converting enzyme inhibitor; ARB: angiotensin II receptor blocker.

- 12.00) vs. 12.00 (12.00 - 12.00) points), or KCL score (4.00 (2.00 - 5.00) vs. 3.00 (2.00 - 5.00) points).

\section{Survey for feasibility of Remote-CR}

After the on-site initial remote system set up, all the patients completed the Remote-CR program session without on-site assistance. The percentage of the response opinions of satisfaction with Remote-CR program, adherence to the exercise or physical activity management are shown in Figure 3. Patients' satisfaction with Remote-CR program was generally high. Remote-CR program helps to maintain unsupervised exercise and physical activity. Most of the patients were walking round their homes and conducted resistance exercise program includ- ing in the exercise video outdoors. Therefore, only about $30 \%$ of the patients performed exercise at home at least three times a week using an exercise video. The initial cost for preparation of the telemedicine system was 520 USD or 440 EUR for a single health provider kit, and 4,260 USD or 3,620 EUR for a single patient kit.

\section{Change in functional status}

We also found no difference in each functional status when comparing the 4-week follow-up in each group. We found no difference in the change in handgrip strength $(3.00(0.00-$ $4.70) \mathrm{kg})$, gait speed $(-0.001(-0.45-0.18) \mathrm{s})$, five-time chair stand test $(-1.01(-2.29-0.09) \mathrm{s})$, and SPPB score $(0.00(0.00$ 
Remote CR was an acceptable way to receive a CR program.

$\begin{array}{lllllllllll}0 \% & 10 \% & 20 \% & 30 \% & 40 \% & 50 \% & 60 \% & 70 \% & 80 \% & 90 \% & 100 \%\end{array}$ remo

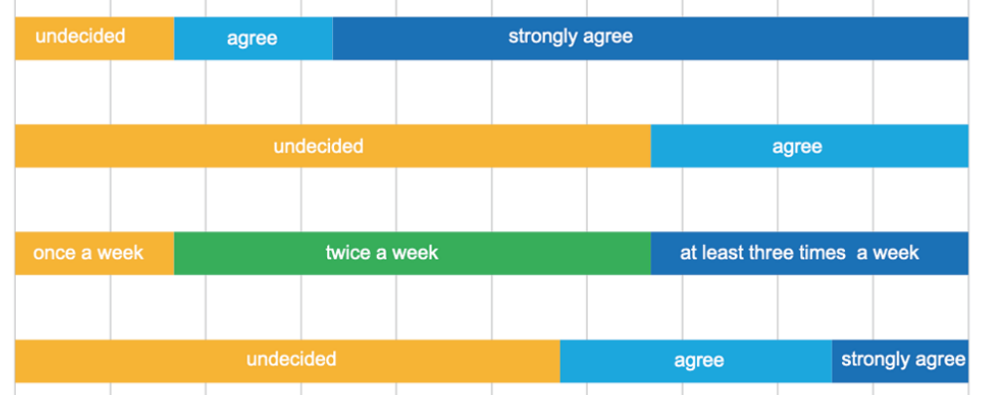

Figure 3. Questionnaire for the satisfaction and adherence to exercise or physical activity management via Remote-CR. RemoteCR: remote cardiac rehabilitation support program.

- 0.00) points) when comparing the Remote-CR and CB-CR, respectively (Table 2).

\section{Cardiovascular-related hospital readmission}

The rate of cardiovascular-related hospital readmission within 6 months after completion of ambulatory CR was comparable (Remote-CR group 0\% vs. CB-CR group 0\%).

\section{Discussion}

To our knowledge, this is the first study to evaluate the feasibility of Remote-CR by a trained physical therapist on adherence of healthy lifestyle behavior, maintaining of functional status and short-term prognosis after completion of $\mathrm{CR}$ in older patients with cardiac disease.
Only $3.8 \%$ of medical institutes in Japan could provide remote CR program as of 2020 [7]. Older subjects have shown to be strongly linked to low digital literacy [17]. Patients' ability to understand how to use digital devices is important to refer a Remote-CR program. However, we included patients with a certain level of digital literacy in this study. To control the selection bias and confounding bias, innovated user-friendly remote real-time monitoring system that can be used by every older patient is essential. All the patients were able to complete the session without on-site assistance. Five out of six patients agree or strongly agree with the item "I feel Remote-CR was an acceptable way to receive a comprehensive CR program". However, there was some feedback from patients about usability or connectivity problems. Improvement of user-friendly system and applications to support continued use would be needed for widespread clinical implementation.

Despite the short follow-up periods, our findings showed a positive effect on the maintenance of functional status, and

Table 2. Within and Between-Group Difference in the Functional Status

\begin{tabular}{|c|c|c|c|c|}
\hline & \multirow{3}{*}{ Baseline, median (IQR) } & \multirow{3}{*}{4 weeks, median (IQR) } & Within-group difference & \multirow{3}{*}{$\begin{array}{l}\text { Between-group difference* } \\
\text { Remote-CR vs. CB-CR } \\
\text { Median (IQR), P-value }\end{array}$} \\
\hline & & & Baseline vs. 4 weeks & \\
\hline & & & P-value & \\
\hline Remote-CR & $11.50(10.75-12.00)$ & $11.50(11.00-12.00)$ & $\mathrm{P}=0.317$ & $0.00(0.00-0.00), P=0.892$ \\
\hline CB-CR & $12.00(12.00-12.00)$ & $12.00(12.00-12.00)$ & $\mathrm{P}=0.317$ & \\
\hline Remote-CR & $27.80(22.75-33.00)$ & $30.50(25.50-34.00)$ & $\mathrm{P}=0.072$ & $3.00(0.00-4.70), P=0.286$ \\
\hline $\mathrm{CB}-\mathrm{CR}$ & $33.00(25.75-34.50)$ & $36.00(26.50-38.00)$ & $\mathrm{P}=0.141$ & \\
\hline \multicolumn{5}{|l|}{ Gait speed } \\
\hline Remote-CR & $3.345(2.303-3.788)$ & $3.220(2.340-3.803)$ & $P=0.686$ & $-0.01(-0.45-0.18), P=0.273$ \\
\hline CB-CR & $3.080(2.980-3.680)$ & $3.030(2.690-3.330)$ & $\mathrm{P}=0.138$ & \\
\hline $\mathrm{CB}-\mathrm{CR}$ & $3.00(2.00-5.00)$ & $2.00(1.00-5.00)$ & $\mathrm{P}=0.680$ & \\
\hline
\end{tabular}

*The change in functional status during intervention periods was determined as the absolute difference and compared the Remote-CR group with CBCR group. IQR: interquartile range; SPPB: short physical performance battery; KCL: Kihon checklist; CR: cardiac rehabilitation; CB: center-based. 
short-term prognosis. The difficulty of maintaining healthy lifestyle behavior and its derived successful ageing has been suggested in older patients with cardiac disease [18]. Caregiver supervision or interaction and self-monitoring and its derived insight in the health status were reported as essential determinants for the long-term healthy lifestyle behavior changes [19]. Thus, we explained to patient's family to participate in the Remote-CR program. All the patients participated in the Remote-CR program with family or caregiver, which would be effective in maintaining the adherence to exercise or physical activity management. We think that one of the advantages of the Remote-CR program is that it involved the family or caregivers into CR program.

Generally, medical cost would be higher for remote CR program; however, it is not covered by National Health Insurance in Japan. Initial cost of telemedicine system demonstrated in this study, 520 USD or 440 EUR for single health provider kit including tablet computer, annual license of the telemedicine system, and 4,260 USD or 3,620 EUR for single patient kit including two tablet computers, electrocardiography, pulse oximeter, electronic sphygmomanometer, and data communication charge. All equipment was prepared by researchers and lent to each patient free of charge. Lack of financial burden may have contributed to satisfaction of Remote-CR program. Future studies should be encouraged to investigate how a cost-efficient Remote-CR program can be implemented.

\section{Limitations}

Several limitations of our present study should be noted. The present study was conducted as case series feasibility study with a small sample size at a single center. The limited number of patients does not allow for a statistical and robust conclusion. The benefit of conducting the Remote-CR on the adherence to healthy lifestyle behavior and patients' functional status, and prognosis should be further investigated with a large sample and long-term intervention. The results of satisfaction of Remote-CR showed the good feasibility of this system. However, the success of the Remote-CR is dependent on the digital literacy and internet networking environment, and application. In the future, we need develop a user-friendly telemedicine system that can be easily used by anyone, anywhere.

\section{Conclusion}

The Remote-CR after completion of ambulatory CR may be applicable as intervention option in selected stable older patients with cardiac disease. The results of this study should prompt further investigation of the usefulness of Remote-CR on functional status, QoL, prognosis, and cost-effectiveness in clinical setting.

\section{Acknowledgments}

The authors would like to thank the cardiac rehabilitation team of Juntendo University Hospital. The authors would also like to thank Mr. Yuji Kobayashi for supporting in setting up and troubleshooting the remote CR system.

\section{Financial Disclosure}

This work is supported by Health, Labour and Welfare Policy Research Grants.

\section{Conflict of Interest}

The authors declare that they have no conflict of interest.

\section{Informed Consent}

All participants gave written consent to participation after explaining the study procedures.

\section{Author Contributions}

TT, TF and HD conceived the study and obtained grant funding. MS, TT, TM and MN-Y contributed to the conception and design of the work. MS, TT, TM, AH, AA and MN-Y contributed to the literature search. MS and TT contributed to the data analysis for the work. MS, TT, TM, NK, and TK contributed to the drafting the manuscript. MS, TT, TM, TM, TA, TF and HD critically revised the manuscript.

\section{Data Availability}

The data supporting the findings of this study are available from the corresponding author upon request.

\section{Abbreviations}

CR: cardiac rehabilitation; QoL: quality of life; CB-CR: center-based CR; remote-CR: remote CR support program; RCT: randomized clinical trial; UMIN: University Hospital Medical Information Network; COVID-19: coronavirus disease 2019; USD: US dollars; EUR: Eros; JACR: Japanese Association of Cardiac Rehabilitation; SPPB: short physical performance battery; IQR: interquartile range

\section{References}

1. JCS/JACR201 Guideline on rehabilitation in patients with cardiovascular disease. https:/www.j-circ.or.jp/cms/ wp-content/uploads/2021/03/JCS2021_Makita.pdf. (Accessed July 20, 2021).

2. Deka P, Pozehl B, Williams MA, Yates B. Adherence to recommended exercise guidelines in patients with heart 
failure. Heart Fail Rev. 2017;22(1):41-53.

3. Frederix I, Vanhees L, Dendale P, Goetschalckx K. A review of telerehabilitation for cardiac patients. J Telemed Telecare. 2015;21(1):45-53.

4. Huang K, Liu W, He D, Huang B, Xiao D, Peng Y, He $\mathrm{Y}$, et al. Telehealth interventions versus center-based cardiac rehabilitation of coronary artery disease: A systematic review and meta-analysis. Eur J Prev Cardiol. 2015;22(8):959-971.

5. Veen EV, Bovendeert JFM, Backx FJG, Huisstede BMA. E-coaching: New future for cardiac rehabilitation? A systematic review. Patient Educ Couns. 2017;100(12):22182230.

6. Rawstorn JC, Gant N, Direito A, Beckmann C, Maddison R. Telehealth exercise-based cardiac rehabilitation: a systematic review and meta-analysis. Heart. 2016;102(15):1183-1192.

7. The report of the cardiac rehabilitation during COVID-19. Data by the Japanese Association of Cardiac Rehabilitation. http://www.jacr.jp/web/ wp-content/uploads/2020/05/COVID-19questionnaire.pdf. Accessed June 2, 2020.

8. Coelho HJJ, Rodrigues B, de Oliveira Goncalves I, Uchida MC. Effects of a short-term detraining period on muscle functionality and cognition of strength-trained older women: a preliminary report. J Exerc Rehabil. 2017;13(5):559-567.

9. Ratel S, Gryson C, Rance M, Penando S, Bonhomme C, Le Ruyet P, Duclos M, et al. Detraining-induced alterations in metabolic and fitness markers after a multicomponent exercise-training program in older men. Appl Physiol Nutr Metab. 2012;37(1):72-79.

10. Marchionni N, Fattirolli F, Fumagalli S, Oldridge N, Del Lungo F, Morosi L, Burgisser C, et al. Improved exercise tolerance and quality of life with cardiac rehabilitation of older patients after myocardial infarction: results of a randomized, controlled trial. Circulation. 2003;107(17):2201-2206.
11. Schulz KF, Altman DG, Moher D, CONSORT Group. CONSORT 2010 statement: updated guidelines for reporting parallel group randomized trials. Ann Intern Med. 2010;152(11):726-732.

12. Guralnik JM, Simonsick EM, Ferrucci L, Glynn RJ, Berkman LF, Blazer DG, Scherr PA, et al. A short physical performance battery assessing lower extremity function: association with self-reported disability and prediction of mortality and nursing home admission. J Gerontol. 1994;49(2):M85-94.

13. Roberts HC, Denison HJ, Martin HJ, Patel HP, Syddall H, Cooper C, Sayer AA. A review of the measurement of grip strength in clinical and epidemiological studies: towards a standardised approach. Age Ageing. 2011;40(4):423429.

14. Collen FM, Wade DT, Bradshaw CM. Mobility after stroke: reliability of measures of impairment and disability. Int Disabil Stud. 1990;12(1):6-9.

15. Sewo Sampaio PY, Sampaio RA, Yamada M, Arai H. Systematic review of the Kihon Checklist: Is it a reliable assessment of frailty? Geriatr Gerontol Int. 2016;16(8):893902.

16. Satake S, Senda K, Hong YJ, Miura H, Endo H, Sakurai T, Kondo I, et al. Validity of the Kihon Checklist for assessing frailty status. Geriatr Gerontol Int. 2016;16(6):709715 .

17. Annual Report on the aging society. Japanese; 2020. https://www8.cao.go.jp/kourei/whitepaper/w-2018/html/ zenbun/s1_3_2_3.html. Accessed December 18, 2021.

18. Schmidt $\overline{\mathrm{IM}}$, Kreutz R, Drager D, Zwillich C, Horter S, Kuhlmey A, Gellert P. Lower Prescription Rates in Centenarians with Heart Failure and Heart Failure and Kidney Disease Combined: Findings from a Longitudinal Cohort Study of Very Old Patients. Drugs Aging. 2018;35(10):907-916.

19. Molloy GJ, Johnston DW, Witham MD. Family caregiving and congestive heart failure. Review and analysis. Eur J Heart Fail. 2005;7(4):592-603. 